\title{
Lidil
}

Revue de linguistique et de didactique des langues

$37 \mid 2008$

Syntaxe et sémantique des prédicats

\section{Le prédicat}

Comment sortir de la Tour de Babel?

\section{Michel Maillard}

\section{CpenEdition}

\section{Journals}

Édition électronique

URL : http://journals.openedition.org/lidil/2686

DOI : 10.4000/lidil.2686

ISSN : 1960-6052

Éditeur

UGA Éditions/Université Grenoble Alpes

\section{Édition imprimée}

Date de publication : 1 juin 2008

Pagination : 23-44

ISBN : 978-2-84310-124-3

ISSN : 1146-6480

Référence électronique

Michel Maillard, «Le prédicat », Lidil [En ligne], 37 | 2008, mis en ligne le 01 septembre 2009, consulté le 19 avril 2019. URL : http://journals.openedition.org/lidil/2686 ; DOI : 10.4000/lidil.2686 


\title{
LE PRÉDICAT
}

\section{COMMENT SORTIR DE LA TOUR DE BABEL?}

\author{
Michel MAILLARD*
}

\begin{abstract}
RÉSUMÉ
À partir des difficultés que présente 1'analyse d'énoncés synonymiques tels que Ce gâteau est délicieux et Délicieux, ce gâteau en termes de «sujet» et «prédicat», cet article se propose de rappeler quelques tournants cruciaux dans l'histoire des deux notions. Le point de départ est le sens de base des termes grecs hypokeímenon et katêgórêma, leur emploi spécifique dans les Catégories d'Aristote, puis leur traduction latine dans les Boetii Commentarii, leur réemploi dans la Grammaire de Port-Royal, et, pour finir, le déplacement conceptuel introduit par J.W. Meiner (1781) et ses disciples, qui voient dans le prédicat l'élément central de la phrase, gouvernant des «arguments» parmi lesquels se trouve le sujet. Le dilemme didactique parait être le suivant: faire le bon choix entre ces conceptions incompatibles de la prédication ou abandonner le prédicat en tant que notion syntaxique.

\section{ABSTRACT}

Synonymic utterances such as Ce gâteau est délicieux and Délicieux, ce gâteau, are difficult to analyse in terms of «subject» and "predicate ». Using this as a starting point, our paper aims at reminding the reader of some crucial turning points in the history of those two notions. We start by looking at the basic meaning of the Greek terms hypokeímenon and katêgórêma, their specific usage in Aristotle's Categories on Interpretation, their latin translation in Boetii Commentarii, their reinvestment in Port-Royal Grammar. Finally, we place emphasis on the conceptual shift introduced by J.W. Meiner (1781) and his followers, who regard the predicate as the central element of the sentence, governing «arguments » amongst which the subject is to be found. The didactic dilemma seems to be the following: either we are forced to choose between these incompatible views on predication, or we give up the idea of the predicate as a syntactic notion.
\end{abstract}

* Professeur émérite de l'université Stendhal 


\section{Petite devinette initiale : où est le prédicat dans Ce gâteau est délicieux?}

Beaucoup de linguistes répondront que le prédicat est constitué par est délicieux, expression verbale complémentaire du sujet ce gâteau, ce qui est conforme au modèle aristotélicien de la proposition. D'autres, minimisant le rôle de la copule à partir de l'existence d'équivalents non verbaux comme délicieux, ce gâteau, diront que le prédicat se réduit à délicieux. $D$ 'autres enfin, invoquant le rôle décisif du verbe comme porteur de la fonction prédicative et centre organisateur de l'énoncé, affirmeront que le prédicat, c'est le verbe être et ils se rapprocheront ainsi des grammairiens allemands et des logiciens de l'école frégéenne. On voit que les réponses varient en fonction des modèles descriptifs dont les linguistes se réclament. Pour l'enseignant, il est clair que le prédicat grammatical ne saurait être à la fois, et sans contradiction, ceci, cela et ça. En didactique des langues, il est donc impératif de faire un choix, à moins qu'une synthèse et une articulation ne soient possibles entre les modèles en présence.

Sujet et prédicat: un couple uni ou en instance de divorce?

La notion de prédicat, fondamentale en logique et en linguistique, a beaucoup évolué au cours de l'histoire de la pensée occidentale, tout comme celle de sujet dont elle est, à bien des égards, complémentaire. Le dogme selon lequel sujet et prédicat seraient dans une relation d'implication réciproque a été remis en question dès la fin du XVIII ${ }^{e}$ siècle mais il a toujours de nombreux adeptes, notamment en France, chez tous les grammairiens qui pensent qu'en français et en anglais le sujet est pareillement obligatoire: dans il pleut comme dans il peut, dans it's raining comme dans he's coming. Cela s'appelle «la servitude subjectale».

\section{Ce que parler veut dire}

Si je «dis $»$ table ou boite, on ne peut pas vraiment dire que je «parle». Je prononce mais je n'énonce pas. En revanche, quand je dis la table boite (sans circonflexe), je fais un 
énoncé, vrai ou faux. Idem si je dis La table est boiteuse. Sans un prolongement verbal, un nom n'est ni vrai ni faux. Le problème de la vérité commence à se poser quand j'accouple un nom avec un verbe pour faire une assertion. Cela, Platon l'avait bien $\mathrm{vu}^{1}$. Selon lui, un énoncé assertif simple - affirmatif ou négatif - comportera donc un nom, désignant ce dont on parle et un verbe exprimant ce qu'on en dit. Après Aristote $^{2}$, le premier sera appelé sujet et le second prédicat. L'opération qui les met en relation recevra, en logique et en linguistique, le nom de prédication.

\section{Toute énonciation n'est pas une prédication}

Pour Aristote, les énoncés modaux - ordres, souhaits, prières - n'entrent pas dans le cadre de la prédication. Il ne s'intéresse qu'aux énoncés assertifs, dans lesquels le locuteur émet un jugement sur quelqu'un ou quelque chose. Les énoncés de ce type sont susceptibles d'être vrais ou faux. Est-il vrai de dire que l'homme est un animal? Peut-on affirmer je suis menteur sans contradiction? L'objet du philosophe est la quête de la vérité, ce n'est pas l'inventaire complet des types d'énoncés. Ainsi une injonction telle que viens! est bien un énoncé, mais comme ce dernier n'est ni vrai ni faux, Aristote ne l'intègre pas à sa logique des prédicats.

\section{Le problème posé par les impersonnels}

Si je dis il pleut, l'énoncé est certes vrai ou faux mais il n'est validable qu'en situation. Or il n'y a de science que du général; Aristote laisse donc de côté les prédicats sans sujet tels

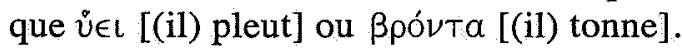

Dès 1985, jugeant que les constructions «impersonnelles » concernaient davantage la question du sujet que celle de la personne, $\mathrm{j}$ 'avais proposé de les rebaptiser asubjectives puis asubjectales (Maillard, 1985 : 64; 1991: 8, 47, 230), car elles peuvent à l'occasion concerner des personnes, y compris la

1. Sophiste, $262 \mathrm{~d}$.

2. Perì Hermēneías, $16 \mathrm{~b} 1$. 
personne de celui qui parle. Dans les exemples (1) et (2) cidessous, les pronoms personnels s'appliquant au locuteur et à ses pairs ne sont pas au cas sujet (nominatif) mais à un cas oblique : datif en (1) (ín âs: à nous) et accusatif en (2) ( $\dot{\epsilon} \mu \epsilon$ : me). Quant au sujet grammatical, il est inexistant:

\begin{tabular}{|c|c|c|c|c|c|}
\hline \multirow[t]{2}{*}{ (1) } & a. & \multicolumn{2}{|l|}{$\dot{\eta} \mu a ̂ a_{S}$} & $\begin{array}{l}\text { пре́ тret } \\
\text { convient }\end{array}$ & 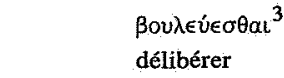 \\
\hline & b. & \multicolumn{4}{|c|}{ «il nous faut délibérer» } \\
\hline \multirow[t]{2}{*}{ (2) } & a. & $\begin{array}{l}\sigma u v e ́ \beta \eta \\
\text { arriva }\end{array}$ & $\begin{array}{l}\dot{\epsilon} \mu \dot{\epsilon} \\
\text { meACC }\end{array}$ & $\begin{array}{l}\phi L \lambda \in \hat{L} \nu \\
\text { aimer }\end{array}$ & 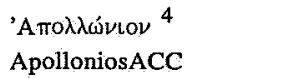 \\
\hline & b. & \multicolumn{4}{|c|}{ «il m'arriva d'aimer Apollonius» } \\
\hline
\end{tabular}

\section{Origine grecque du couple sujet/prédicat}

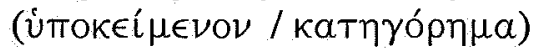

Venues de l'univers de la parole ordinaire, en grec comme en latin, les notions de «sujet» (gr. hypokeimenon, lat. subjectum, «ce qui est mis en-dessous») et de «prédicat» (lat. praedātum «ce qui est proclamé par le praeco, crieur public», gr. katēgórēma «accusation» puis «jugement» et enfin «prédicat») se sont spécifiées et restreintes dans la sphère de la logique aristotélicienne en perdant leurs connotations défavorables - le sujet étant simplement «ce dont on parle» (en bien ou en mal) et le prédicat «ce qu'on en dit» (vrai ou faux, selon les cas).

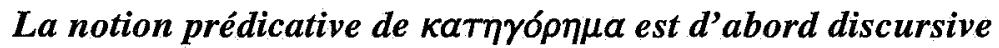

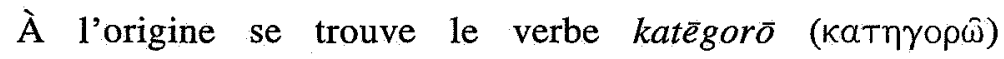
«accuser». Le nom de l'agent est katēoros (katńyopos) «accusateur», celui du patient katēgoroúmenos

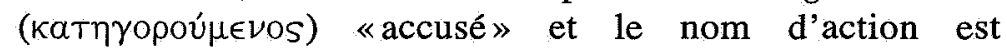

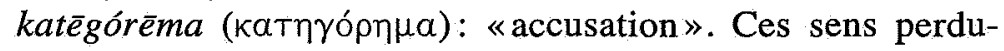
rent en néo-hellénique, parallèlement à l'usage grammatical

3. Thucydide, cité par Lambert (2006: 7).

4. Apollonios Dyscole (1997, Syntaxe, 3, 345, 87). 


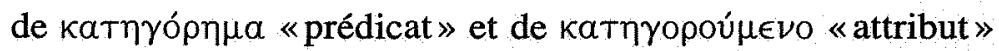
(inclus dans le prédicat), qui attestent un effet en retour de la grammaire latine, elle-même inspirée de la logique aristotélicienne.

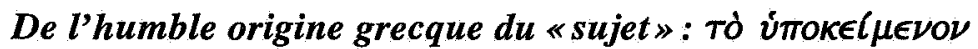

Je voudrais insister sur la condition «assujettie» de l'ancêtre anthropomorphe de ce sujet grammatical dont les enseignants disent encore trop souvent qu' «il fait l'action», ce qui est le

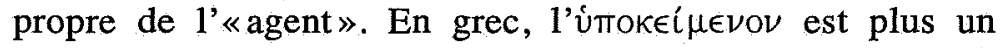
«patient» qu'un agent. Il est «soumis» aux lois de la vie et à celles de la cité, auxquelles il est parfois accusé de contrevenir. Aujourd'hui encore, l'vток $\in$ í $\mu \in \nu$ o [ipokímeno] est un «mauvais sujet» habitant les bas-quartiers.

Devenu abstrait dans le métalangage philosophique, le «sujet», couché sous le discours, peut être provisoirement caché par lui. C'est à partir des prédicats qui déclinent ses propriétés que l'úToкєí $\mu \in \nu O \nu$ sort peu à peu de l'ombre.

\section{Le support de validation aristotélicien ne se réduit ni au sujet ni au thème}

L'erreur des épigones d'Aristote est d'avoir réduit

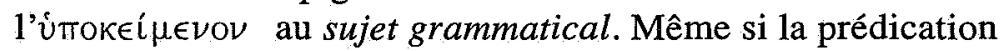
aristotélicienne est souvent illustrée par des énoncés de type SN-SV, le support de validation peut se présenter sous d'autres formes que celle d'un sujet, à savoir celle d'un datif ou d'un autre cas oblique. D'ailleurs, en grec ancien, le pluriel Tà $\dot{\tau} \pi о \kappa \in \dot{L} \mu \in \nu a$ ne désigne-t-il pas aussi «les circonstances » qui conditionnent la validité d'une affirmation ?

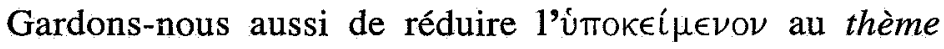
$(\theta \epsilon ́ \mu a)$, car celui-ci a été préalablement posé, alors que celuilà peut être nouveau (rhématique) comme dans l'énoncé

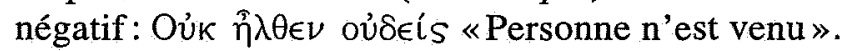

De même, il est difficile d'assimiler à un thème le sujet de l'énoncé Qui est venu? puisque rien n'a été dit dans le discours sur le référent de qui, dont l'existence reste problématique. Malgré la qualité de son argumentation, je ne puis donc m'associer vraiment à David Gaatone quand il propose de 
substituer à l'opposition sujet/prédicat le couple thème/rhème (voir Gaatone, ici-même), qui ne se situe pas sur le même plan logico-grammatical.

\section{L'apport original des Stoïciens et leur modèle verbo-centré}

$\mathrm{Si}$, pour Platon, en-deçà de la combinaison nom + verbe (óvo $\mu \alpha+\rho \hat{\mu} \mu \alpha)$, il n'y a pas d'énoncé du tout, pour les Stoïciens, en revanche, un verbe assertif à lui seul peut constituer un énoncé. Ainsi que le rappelle M. Baratin: «le prédicat n'est plus, dans la conception stoïcienne, un constituant qu'il faudrait considérer sur le même plan que l'autre, le sujet, comme le fait Aristote. Au contraire, le prédicat est le noyau et comme l'essence même de l'énoncé [...], ce qui occupe la fonction de sujet n'est que la condition de sa vérification"

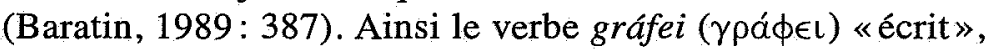
sans sujet ni objet exprimé, est un énoncé logiquement incomplet qui appelle la question qui ? et sans doute aussi la question quoi? mais ce n'en est pas moins un énoncé, un lektón $\left(\lambda \in \kappa\right.$ Tó $\nu$ ), quelque chose qui peut se dire...et qui se $\mathrm{dit}^{5}$.

Mieux, un verbe à lui seul peut constituer un énoncé complet si le nom est compris dans le verbe, donc si ce dernier équivaut à une combinaison nom + verbe (Desbordes, 1991 : 14). Certes les Stö̈ciens ne parlent pas des impersonnels atmosphériques car, pour la majorité des Grecs, les turbulences de l'air sont gérées par la personne de Zeus. Il est pour-

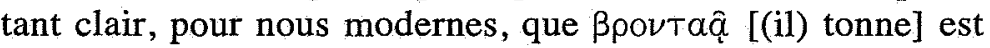
un prédicat qui se passe de tout sujet, y compris de Bpovtń «tonnerre», qui ferait redondance, puisque ce nom est

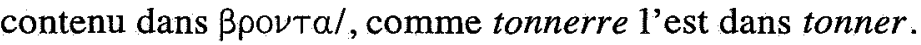

Les Stoïciens ont eu aussi le mérite de reconnaitre l'existence d'autres prédicats impersonnels dont le support logique de prédication apparait à un cas oblique, comme dans un énoncé cité par Desbordes (1991: 18), Sōkrátei mélei [Pour

5. Cf. Ildefonse (1997: 146), se référant à Diogène Laerte. 
Socrate il y a un objet de souci], comprenons « Socrate se fait du souci». Si, dans la «bonne» traduction française, Socrate est en position de sujet, en grec, par contre, il est en position de complément. Sōkrátei est un datif dans une structure que nous dirions aujourd'hui expériencielle puisque l'actant marqué [+ humain], l'expérient de G. Lazard, fait l'expérience du souci sans être pour autant représenté syntaxiquement par un sujet grammatical. En vérité, mélei est un impersonnel qui exclut tout sujet, un verbe monovalent dont le seul actant n'est pas le prime actant de Tesnière.

On a des constructions expérientielles analogues en allemand et en russe mais il a fallu attendre le XVIII' siècle, notamment avec Miklosich (1883), pour que soit reconnue officiellement la présence de prédicats sans sujet dans les langues slaves et germaniques - all. mich friert [me fait froid] ou russe menja znobit [me frissonne]. Leur existence en français est plus difficile à admettre car beaucoup tiennent le $i l$ de $i l$ me semble ou de il me faut pour un sujet. Comme Berrendonner (par ex. 2000: 50) et moi-même l'avons souvent répété, il n'est pas un sujet, c'est un flexif d'avant, inhérent au prédicat, qui se borne à marquer le statut délocutif de la forme verbale.

\section{L'importance de Boèce dans la transmission de I'héritage grec}

Philosophe et politique latin du $\mathrm{VI}^{\mathrm{e}}$ siècle, Boèce (lat. Boetius), après avoir achevé ses études à l'École d'Athènes, fut nommé consul sous Théodoric. Accusé de complot, il fut emprisonné. Il écrivit alors ses Commentaires sur Aristote, qu'il traduisit en latin. Héritier de la culture grecque, il souhaitait la transmettre au monde occidental. Cela dit, il a davantage contribué à diffuser la logique aristotélicienne que celle des Stoïciens, injustement oubliés pendant des siècles.

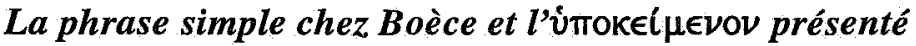 comme subjectus}

«Est enim simplex oratio quae duobus terminis constat. Termini autem sunt nomina et verba, quae in simplici proposi- 
tione praedicamus, ut in eo quod est Socrates disputat, Socrates et disputat termini sunt ${ }^{6} \gg$ [La phrase simple est celle qui comporte deux termes. Ceux-ci sont les noms et les verbes que nous mettons en relation prédicative, comme dans l'exemple Socrate discute, où Socrate et discute constituent les termes]. L'auteur poursuit «Et qui minor terminus in enuntiatione proponitur, ut Socrates, subjectus dicitur et ponitur prior» [Et le terme mineur proposé dans l'énonciation, tel Socrates, on l'appelle sujet et on le place devant].

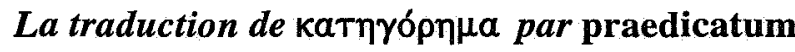

Suit la présentation du prédicat sous la forme du verbe praedicare «énoncer»: «qui vero maior, praedicatur et locatur posterior, ut disputat» [celui qui est en vérité le plus important, on l'énonce et on le place après, tel disputat]. Mais on voit aussi, chez Boèce, apparaitre le substantif praedicatum: «Has vero enuntiationum partes, id est praedicatum atque subjectum terminos appellamus ${ }^{7} \gg$. [Ces parties d'énoncés, c'est-à-dire le prédicat et le sujet, nous les appelons termes].

\section{Le modèle logico-grammatical des grammairiens} de Port-Royal

Arnauld et Nicole réactualisent, dans leur Logique de 1662, le modèle Subjectum/Praedicatum: "Ce jugement s'appelle aussi proposition, et il est aisé de voir qu'elle doit avoir deux termes: l'un, de qui l'on affirme, ou de qui l'on nie, lequel on appelle sujet; et l'autre que l'on affirme, ou que l'on nie, lequel s'appelle attribut ou praedicatum ${ }^{8} \gg$.

Dans leur grammaire, Arnauld et Lancelot introduisent un nouveau terme, la copule, et remplacent Prédicat par Attribut, installant durablement ce dernier terme en position prédica-

6. Commentarii, Pars Prior, 77, cité par G. Graffi (2006: 1).

7. Introd. ad syll. Categ., PL 64, 768D, cité également par Graffi (2006:1)

8. Arnauld et Nicole (1662, II : 3, 182) cités par Graffi (2006:1). 
tive dans le métalangage français. Partant de La terre est ronde, ils généralisent en déclarant: «[...] toute proposition enferme nécessairement deux termes. L'un appelé sujet, qui est ce dont on affirme, comme terre, et l'autre appelé attribut, qui est ce qu'on affirme, comme ronde; et de plus la liaison entre ces deux termes, est ». (Arnault et Lancelot, 1660, II : 1,23)

\section{Impuissance du modèle de Port-Royal à rendre compte des impersonnels}

Bien que ce modèle grammatical, diffusé en Occident pendant plus de trois siècles et réactualisé par les chomskiens, accorde une juste place au rôle assertif et relationnel du verbe-copule, il ne distingue pas suffisamment prédication logique et prédication grammaticale.

S'il est vrai que toute proposition au sens d'Aristote exige pour être validée un support logique de référence - qu'on 1'appelle ن́токєí $\mu \in \nu$ $\nu$, subjectum ou autrement - il est faux que toute proposition, au sens linguistique du terme, contienne ce support sous la forme d'un sujet grammatical. Beaucoup d'énoncés attestés dans les langues du monde font infraction à cette «loi », entre autres l'impersonnel italien $s i$ fa tardi «(il) se fait tard», que les auteurs connaissaient bien et qui exclut visiblement tout sujet. (id. II, 9, 88)

\section{Le cas de l'impersonnel il est six heures}

Ce n'est pas dans le passage consacré aux impersonnels qu'Arnauld et Lancelot abordent l'énoncé Il est six heures qui ne correspond en rien à leur schéma prédicatif. C'est, assez hypocritement, dans la dernière partie de l'ouvrage, réservée aux «figures de construction». Le problème, c'est que l'indication de l'heure n'a rien d'une «figure». C'est un énoncé aussi banal que l'anglais $I t$ 's six o'clock. Selon les auteurs, «[...] il faudrait dire elles sont six heures, comme on le disait autrefois [...]» (id. XXIV, 107). A-t-on jamais parlé ainsi ? Les langues romanes du sud maintiennent, il est vrai, l'accord du verbe «être » avec son groupe nominal postposé. C'est le cas du portugais qui dit são seis horas [*sont 
6 heures]. Mais la construction française, elle, est bien impersonnelle et il convient de la prendre comme telle. Aujourd'hui comme hier, ne rit-on pas des enfants ou des étrangers qui demandent $*$ Quelle heure est-elle ${ }^{9}$ ou Quelles heures sontelles?

\section{Le modèle verbocentrique allemand et son expansion en Europe}

C'est en Allemagne, à la fin du XvIrre siècle, que se fait jour l'idée nouvelle que le verbe assume une fonction propre, la fonction prédicative, et qu'il constitue le centre organisateur de la phrase. Dans cette perspective, le sujet cesse de peser d'un poids égal au verbe dans la balance syntaxique. Il devient une sorte de dépendance du verbe, un complément comparable aux autres. La structure propre de la langue allemande, où les impersonnels sont fréquents et où le verbe principal tient un rôle de pivot autour duquel tournent les éléments nominaux, n'a pas peu aidé les grammairiens et les logiciens à se dégager du modèle français issu d'Aristote.

\section{Le modèle logico-grammatical de Johann Werner Meiner (1781)}

Tout comme en France, la pensée grammaticale allemande se nourrit au XVIII de réflexion philosophique et les grammairiens y sont aussi des logiciens. C'est le cas du professeur de langues, J. W. Meiner, disciple de Leibniz, qui écrit aussi des ouvrages de logique et une grammaire philosophique d'inspiration logicienne (voir Meiner, 1781).

Le prédicat s'y trouve établi comme centre conceptuel de la proposition. Sujet et compléments ne sont plus alors que des «déterminations» du prédicat, appelées par celui-ci chaque fois que l'exige sa complétude sémantique.

9. Un de nos relecteurs nous rappelle, à juste titre, que le passage au féminin dans ce type d'énoncé a été une proposition (humoristique ?) de réforme linguistique portée par le mouvement féministe après 1968. 


\section{La diffusion des modèles verbocentriques au $\mathrm{XIX}^{e}$ et au $X^{e}$ siècles}

Les représentants allemands de cette tendance issue de Meiner sont surtout Herling (1830), Becker (1836) et Götzinger (1836). Tous trois sont des disciples de Humboldt, notamment le médecin Becker, qui l'a connu personnellement.

S'inscrit dans la même perspective verbocentriste le mathématicien Frege (voir Frege, 1891), fondateur de la logique moderne, chez qui la prédication est analysée comme une relation entre un prédicat - devenu siège de la fonction propositionnelle $f$ - et un nombre $n$ de termes définis ou arguments, le tout représentable en grammaire par $\mathrm{P}(\mathrm{x})$ pour les prédicats à 1 place, $\mathrm{P}(\mathrm{x}, \mathrm{y})$ pour les prédicats à 2 places, $\mathrm{P}(\mathrm{x}$, $\mathrm{y}, \mathrm{z})$ pour ceux à 3 places.

Le Portugais Coelho (1881), le Russe Dmitrievskij (1877) (Sériot, 2004), 1'Ukrainien Potebnja (1888) (Sériot, 2002) et le Français Tesnière (1959) se rattachent à ce courant verbocentriste. On retrouve une même orientation aujourd'hui chez Creissels.

\section{Approche de la notion de Prédicat dans Creissels (2006)}

«En logique, un prédicat est une expression simple ou complexe qui donne naissance à des énoncés assertifs (susceptibles d'être jugés vrais ou faux selon la situation de référence à laquelle on les applique) en se combinant avec des arguments qui représentent des entités [...]» (Creissels, 2006, I: 39). Et, plus loin, «Il y a une similitude évidente entre la combinaison logique d'un prédicat avec ses arguments et la formation d'une unité phrastique par combinaison d'un verbe et d'un certain nombre de constituants nominaux. Par analogie avec la combinatoire logique prédicats-arguments, on désigne couramment comme arguments du verbe les entités dont le verbe implique la participation" (id.). Il est clair qu'une telle approche se situe dans la perspective frégéenne que nous avons définie supra. 


\section{Le problème des "prédications non verbales »}

Si le syntacticien se donne comme outil descriptif un modèle verbo-centré, qui tend à identifier le prédicat au seul verbe, comment abordera-t-il le problème des phrases averbales?

À propos d'un énoncé français comme Délicieux, ton gâteau, le linguiste verbocentriste peut être tenté de le présenter comme un énoncé «syntaxiquement déficient» (id., I, 344) en montrant que, faute de verbe, il ne peut être mis en subordination:

* Je dis que délicieux ton gâteau est récrit $J e$ dis que ton gâteau est délicieux.

Cela permet de récupérer la copule manquante et de retrouver la phrase canonique de base, mais tous les adeptes du modèle verbo-centré ne sont pas prêts à accepter cette transformation. C'est le cas de Blanche-Benveniste. (2004, p. 2 de la prépublication)

\section{La notion de "nexus» chez \\ Claire Blanche-Benveniste (2004)}

Dans une communication faite à l'université de Provence au cours du Colloque sur la Prédication (4-6 nov. 2004), Claire Blanche-Benveniste aborde le problème des «prédications sans verbe», telles qu'on en rencontre, par exemple, dans les catalogues de vente par correspondance: Vos carrelages remis à neuf - Votre table bien protégée - Réfrigérateur propre en un clin d'ceil ${ }^{10}$.

En s'inspirant d'Eriksson (1993), qui a lui-même puisé son inspiration chez Jespersen (1924), C. Blanche-Benveniste utilise le terme de nexus, pour couvrir les divers types de "prédication sans verbe $»$ en français. Voici comment Eriksson (1993: 26) définissait la notion: «Nous appellerons nexus l'unité syntaxique qui résulte d'une prédication assurée par une unité autre que le syntagme verbal».

10. Exemple 60 de la prépublication. 
Tout en retenant la notion de nexus, l'auteur écrit, à propos des prédications averbales en général: "Il s'agit de savoir si le terme de prédication a ici la même valeur que pour la prédication assurée par un verbe tensé et si on peut postuler qu'elle a un sujet. C'est un peu ce que semblait dire Jesper$\operatorname{sen}^{11}$ et c'est en gros ce qui a été proposé ensuite par les analyses en «small clauses ${ }^{12} »$ de la grammaire générative» (C. Blanche-Benveniste, 2004, p.2 de la prépublication). Et l'auteur de poursuivre: «Il me semble peu rentable de projeter des notions verbales (prédication, sujet) sur des organisations syntaxiques dépourvues de verbe. C'est certainement très utile dans les paraphrases, afin de mieux expliciter l'interprétation en nexus. [...]. Mais expliciter et analyser sont deux choses différentes. Le rétablissement d'une prédication explicite, très utile pour assurer une lecture sémantique, risque de changer la structure syntaxique étudiée, de réduire à un seul type des organisations qui peuvent relever de types différents et d'introduire du matériel supplémentaire dont il est ensuite difficile d'expliquer la disparition. Je m'efforcerai de ne pas utiliser les paraphrases comme éléments de l'analyse syntaxique ». (id., 2-3)

Méfiante à l'égard des tentations réductionnistes de certains linguistes - et notamment des chomskiens - 1'auteur fait aussi quelques propositions métalinguistiques: «Réduire ces différents types à un seul et même modèle, comme il a été proposé de le faire en utilisant les désignations de sujet et prédicat, n'est sans doute pas nécessaire. On pourrait tout aussi bien les désigner comme des relations entre des supports et des apports, ce qui aurait l'avantage de ne pas engager l'analyse dans des analogies risquées avec les constructions verbales ». (id., conclusion)

Il est intéressant de souligner que la dimension temporelle n'est pas absente de ces énoncés sans verbe, pas plus que la dimension modale. Lorsqu'un locuteur s'adressant à un gar-

11. «Combinaison entre deux mots ou groupes de mots qui entretiennent une relation sujet-prédicat» (Jespersen, 1924, cité par C. BlancheBenveniste).

12. Cardinaletti and Guasti (1995), M.A. Jones (1996). 
çon de restaurant ordonne: moins cuites, mes pâtes, la prochaine fois! il est clair que mes pâtes constitue le «support» sur lequel vient s'appliquer un «apport» modal et temporel moins cuites [...] la prochaine fois.

\section{En arabe, phrases verbales et averbales sont en opposition radicale}

Contrairement à ce qui se passe pour le français, la «phrase nominale» de l'arabe ne peut être récrite avec une copule verbale :

(3) a.

$$
\text { mohmmad-un }
$$

maīd-un

b. «Mohammed (est) malade»

$$
\text { mohammed-NOM. MSG malade-NOM. MSG }
$$

L'énoncé attribue au sujet logique de la prédication, mohmmadun, une propriété actuelle. Le nom propre est au nominatif masc. sing. et il en va de même pour l'adjectif prédicatif mariđ̋n, proche de notre «attribut» français. Nom et qualificatif sont accordés en nombre, genre et cas. Il n'est pas question d'imaginer ici, comme on le fait souvent pour les énoncés non verbaux du français, une ellipse de la copule, l'arabe ne disposant pas, pour le présent, d'un verbe comparable à «être».

En revanche, ce dernier apparaît au passé, pour marquer le temps. Il figure en tête de phrase - comme un verbe quelconque - suivant le schéma classique VSO ou VSC (où $O$ signifie «objet» et $\mathrm{C}$, de manière plus extensive, «complément»):

(4) a. kạ̄na mohmmad-un

marid-an

était mohammed-NOM.MSG

malade-ACC.MSG-

b. «Mohammed était malade»

L'《attribut» passe à l'accusatif, comme s'il était le complément d'un verbe ordinaire - et même un complément d'objet - ce qui est loin des usages du latin et du grec, où l'attribut du sujet est au même cas que le sujet (au nominatif). 


\section{En polonais, la prédication peut s'appuyer sur une copule non verbale}

Il en va ainsi dans l'énoncé suivant, où le rôle de la copule est tenu par to, un pronom démonstratif neutre, parfois proche de notre ça, comme dans la question co to ? [(c'est) quoi, ça ?] ou l'affirmation to prawda [ça, (c'est) la vérité]:

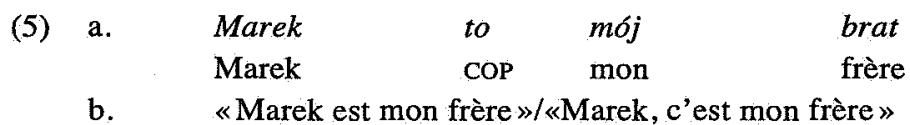

Le polonais dit à peu près [*Marek /ça/ mon frère], comme le franco-créole des Seychelles dit Marek sa zom [*Marek /ça/ homme] «Marek est un homme», avec une copule issue du ça français. Utiliser un pronom comme copule n'est pas rare dans les langues. On retrouve cet usage en hébreu et en arabe, ainsi que dans de nombreux autres idiomes.

\section{Articuler les deux modèles sans contradiction}

En arabe, où «phrase nominale» et «phrase verbale» obéissent à des règles totalement différentes, il n'est pas trop de deux modèles pour en rendre compte: l'un en Support/Apport, inspiré du schéma $S / P$ d'Aristote pour la phrase nominale, 1'autre en $P\left(C_{0}, C_{1}, C_{2}\right)$ de type frégéen, où tous les arguments nominaux - y compris le sujet $\mathrm{C}_{0}-$ sont vus comme des compléments du verbe.

En français, 1'opposition entre les phrases canoniques dites «verbales» (Ève enseigne la linguistique) et les phrases canoniques dites «nominales» (Ève est enseignante de linguistique) n'est pas aussi tranchée qu'en arabe. D'où la possibilité d'établir une équivalence entre un prédicat composé d'une copule et d'un attribut (est + enseignante de linguistique) et un prédicat composé d'un verbe et d'un complément (enseigne + la linguistique). Comme cette équivalence, inapplicable en arabe, n'est pas universelle, il est bon de garder en réserve les deux modèles historiques de la proposition pour les faire jouer complémentairement, y compris en français. 
Sur ce point, nous sommes d'accord avec Sériot (2006) ${ }^{13}$.

\section{Suggestions aux enseignants}

Devant un énoncé prédicatif averbal, on pourra continuer à faire appel au modèle aristotélicien en $\mathrm{S} / \mathrm{P}$, non sans modifier quelque peu la terminologie traditionnelle, selon les suggestions de Claire Blanche-Benveniste, (cf. supra) en distinguant un «Support de prédication» et un «Apport prédicatif». Issu du modèle $S / P$, le modèle $S / A$ admet une permutation entre $S$ et $\mathrm{A}$, si nécessaire, comme dans le portugais exquisito, aquele casal ou le français bizarre, ce couple-là dans lesquels A précède $\mathrm{S}$. Ajoutons que $\mathrm{A}$ peut s'employer sans $\mathrm{S}$ - exquisito! «bizarre»-le support logique de validation (l'hypokeímenon d'Aristote) se situant seulement dans le contexte. N'oublions pas qu'eñ grec les hypokeímena désignent aussi les «circonstances » qui permettent de valider un énoncé. Bien qu'isolé, l'adjectif constitue bien un «apport prédicatif».

$\mathrm{Si}$ la construction est verbale, et qu'on a un «prédicat à 3 places », le modèle verbo-centré d'origine frégéenne de type $\mathrm{P}\left(\mathrm{C}_{0}, \mathrm{C}_{1}, \mathrm{C}_{2}\right)$ convient très bien. L'ancien «sujet», devenu $\mathrm{C}_{0}$, $\mathrm{y}$ apparait comme le complément d'origine tandis que $\mathrm{C}_{1}$ et $\mathrm{C}_{2}$ désignent les autres compléments du prédicat qui ont statut d'arguments (par exemple l'objet premier et l'objet second). Si le verbe ne se suffit pas à lui-même comme dans le cas de $a$ volé, il appelle une triple complémentation, liée aux 3 questions: qui? (pour le complément d'origine), quoi? (pour le complément d'objet premier), à qui ? (pour le complément d'objet second). Par exemple Paul a volé une bille à Pierre. Ici Pierre est «l'objet d'un vol» (objet second) et la bille est «l'objet volé» (objet premier) ${ }^{14}$.

13. Voir Sériot (2006): table ronde du colloque La Structure de la proposition.

14. Bannir le «complément d'objet second» pour revenir au vieux «complément d'attribution», comme le veut le rapport Bentolila, nous parait une décision bien mal inspirée: qu'est-ce que le voleur peut bien «attribuer» au volé ? La notion d'attribution convient mieux aux donations qu'aux spoliations. Certes dans les grammaires de cas, la notion de «bénéficiaire» inclut les «bénéficiaires négatifs» mais le concept d'《objet second» a, entre autres mérites, celui d'être neutre quant à l'opposition bénéficiaire/détrimentaire. 
Voyons maintenant ce qui se passe lorsque le prédicat verbal se suffit à lui-même, comme il advient en portugais avec amanhece «le jour se lève», qui contient en lui-même tout ce qui est nécessaire à sa compréhension et, partant, n'appelle aucun complément d'information. Dans ce cas, $\mathrm{C}_{0}$ et $\mathrm{C}_{1} \mathbf{n}$ 'ont plus lieu d'être. Le verbe est de valence zéro, il est «avalent» comme dirait Tesnière (1959: 239). Et dans le français $i l$ faitjour? Dira-t-on que $i l$ est le sujet de fait ? ${ }^{15}$. Allons-nous demander qui fait jour? On sait que c'est la rotation de la terre sur elle-même qui fait le jour et la nuit, mais la langue n'en a cure.

\section{Qu'est-ce que la «servitude subjectale » du français?}

Selon nous, elle signifie non pas que le sujet y serait obligatoire en toutes circonstances - ce qui n'existe dans aucune langue - mais qu'en l'absence d'un sujet nominal, le verbe doit être accompagné d'un indice de sujet (Tesnière, $1959,132,134,137-143)$ qui lui est affixé. Il peut ne répond pas à la question Qui peut? mais fait rebondir la question du sujet: Qui il? Le il de il peut n'est pas plus le sujet de peut que ne l'est la désinence du latin potest ou celle du portugais pode. L'indice de sujet n'est rien qu'une marque sur le prédicat qui nous oriente vers la recherche du sujet caché (hypokeímenon, subjectum)...à supposer que le verbe en réclame un. La question * Qui pleut? - encore possible chez Aristophane ${ }^{16}$ - a cessé depuis longtemps de se poser. L'indice de sujet français s'est vidé ici de toute référence et ne renvoie plus à rien ni à personne. Idem pour il-fait-jour où nul ne peut répondre à la question qui fait quoi ? Il en va de même avec $\mathrm{Il}$ en-va-de-même - qu'on nous pardonne cette écholalie ! - où $i l$ et $e n$ sont aussi vides l'un que l'autre. Ces constructions lexicalisées ne se laissent plus analyser en sujet/prédicat. Elles constituent à elles seules un prédicat. Il faut les comprendre

15. On aimerait connaitre sur ce point la position des co-auteurs du rapport Bentolila pour qui le sujet «fait ou subit l'action exprimée par le verbe » (IV, 1, p. 18) ... comme disait mon grand-père.

16. Les Nuées, 367-381. 
globalement - comme veulent le suggérer nos traits d'union. Elles sont impersonnelles, ou mieux, asubjectales.

\section{En réponse à la devinette initiale}

Dans ce gâteau est délicieux, il est exclu pour nous de présenter délicieux comme le prédicat. Nous dirons que c'est un adjectif en position prédicative (angl. predicative, port. predicativo), par opposition au statut épithétique qu'il a dans ce gâteau délicieux ou ce délicieux gâteau.

Nous ne dirons pas non plus que est constitue le prédicat de la phrase car on a intérêt à conserver pour le français l'idée d'un prédicat constitué de copule + prédicatif (attribut) en vertu d'équivalences prototypiques telles que: est enseignant $\approx$ enseigne, devient vieux $\approx$ vieillit, devient jaune $\approx$ jaunit etc. Ici le prédicat est constitué des deux termes est délicieux.

Dans la «phrase nominale» délicieux, ce gâteau, le lien prédicatif entre les deux termes est de nature non verbale. Ce qui remplace la copule verbale, c'est ce qu'on pourrait appeler une pause copulative, ce bref silence qui disjoint 1'adjectif du substantif et empêche d'interpréter délicieux comme épithète de ce gâteau. L'important est d'éviter toute confusion entre l'énoncé délicieux, ce gâteau et le SN ce délicieux gâteau.

Outre la pause, l'ordre des mots est fonctionnel: grâce à l'antéposition de l'adjectif, le déterminant $c e$, qui se trouve placé entre délicieux et gâteau, contribue à disjoindre adjectif et substantif en achevant de casser tout lien épithétique entre eux. Pour éviter une possible confusion entre prédication verbale et prédication non verbale, nous appellerons cette dernière «nexus», en suivant l'heureuse suggestion de Claire Blanche-Benveniste, et nous dirons que délicieux est un «apport» dont ce gâteau est le «support».

Cet énoncé averbal, qui peut être vrai ou faux, intéresse la logique des prédicats au même degré que l'énoncé canonique ce gâteau est délicieux. Pour le logicien, la problématique est la même, seule diffère la présentation. Pour le grammairien, 
délicieux, ce gâteau, ne saurait comporter de sujet grammatical puisqu'il n'y a pas de verbe dont ce gâteau pourrait être sujet. En revanche, la structure apport/support convient très bien pour décrire un tel type d'énoncé.

\section{RÉFÉRENCES BIBLIOGRAPHIQUES}

Apollonius Dyscole (éd. Lallot) (1997): De la construction

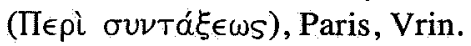

ARNAULD, A. \& C. LANCELOT [1660] (1969): Grammaire générale et raisonnée, Paris, Repr. Paulet.

BARATIN, M. (1989) : La naissance de la grammaire à Rome, Paris, Minuit.

BECKER, K. F. (1836-1839): Ausführliche deutsche Grammatik als Kommentar der Schulgrammatik. [...], Francfort-sur-le-Main, Johann Christian Hermann'sche Buchlandlung, G. F. Kettembeil.

Bentolila, A., Desmarchelier, D., Orsenna, E. (2006) : Rapport de mission sur l'enseignement de la grammaire, Paris, Univ. Paris V et Académie française (version internet).

BERRENDONNER, A. (2000): Que reste-t-il de nos actants? Les passifs impersonnels en français, in P. Sériot \& A. Berrendonner (dir.) Le paradoxe du sujet. Les propositions impersonnelles dans les langues slaves et romanes/Cahiers de l'ILSL, 12, Lausanne, Unil, 43-53.

BLANCHE-BENVENISTE, C. (2004): Les nexus nominaux, à paraitre in Actes du colloque sur la Prédication, (Univ. de Provence, 4-6 nov. 2004).

Boece (Boetius) (1877-1880): A. M. S. Boetii Commentarii in librum Aristotelis IEPI EPMHNEIA $\Sigma$, recensuit C. Meiser, Lipsiae, in aedibus B. G. Teubneri.

Cardinaletti, A. \& M. J. GuAsti (eds.) (1995): Small Clauses, New York, Academic Press.

Chocheyras, J. (éd.) (1985): Autour de l'impersonnel, Grenoble, Ellug.

COOKE, H. P. (éd.) (1983): Aristotle: The Categories on Interpretation, Cambridge Massachusetts, Harvard University Press.

CREISSELS, D. (2006): Syntaxe générale, une introduction typologique, Paris-Cachan, Hermès Lavoisier (2 vol.).

DESBORDES, F. (1991): L'impersonnel d'après les textes théoriques de l'Antiquité, in M. Maillard (éd), 11-18. 
ERIKSSON, O. (1993): La phrase française. Essai d'inventaire de ses constituants syntaxiques, Göteborg, Acta Universitatis Gothoburgensis.

FREGE, G. [1891-1892] (1994): Funktion, Begriff, Bedeutung, Göttingen, Vandenhoeck \& Ruprecht.

GaAtone, D. (1999): Les phrases à sujet postiche, in Mélanges de linguistique, sémiotique et narratologie dédiés à la mémoire de Krassimir Mantchev à l'occasion de son $60^{\circ}$ anniversaire, Sofia, Colibri, 122-138.

GöTZINGER, M. W. (1836-1839): Die deutsche Sprache, Stuttgart, Hoffmann.

GrafFI, G. (2006): Subjectum et Praedicatum de l'antiquité classique à Port-Royal, à paraitre in Actes du Colloque sur la structure de la proposition, (Univ. de Lausanne, 5-7 oct. 2006).

Herling, S. H. A. (1830-1832): Die Syntax der deutschen Sprache [...]. Francfort sur le Main, Johann Christian Hermann'sche Buchhandlung, G.F. Kettembeil.

JESPERSEN, O. [1924] (1968): The Philosophy of Grammar, London, Allen and Unwin.

Jones, M. A. (1996): Foundations of French Syntax, Cambridge, Cambridge University Press.

LAMBERT, F. (2006) : Peut-on parler d'impersonnel en grec ancien? à paraitre in Actes du colloque sur l'Impersonnel, Univ. d'Angers, 8-9 déc. 2006.

LAZARD G. (1994): L'actant $\mathrm{H}$ : sujet ou objet? Bulletin de la Société de linguistique de Paris, 89, 1-28.

LAZARD G. (2004): La prédication implique-t-elle un sujet? à paraitre in Actes du colloque sur la Prédication (Université de Provence, 4-6 nov. 2004).

MAILlARD, M. (1985): L'impersonnel français de il à $f a$, in Chocheyras (éd.), 63-118.

MAILLARD, (éd.) (1991): L'impersonnel, Grenoble, Ceditel.

MAILLARD, (éd.) (1994): L'impersonnel, L'Information grammaticale, 62 .

MAILlARD, (2004): Y a-t-il prédication sans sujet ni verbe? à paraitre in Actes du colloque sur la Prédication, (Univ. de Provence, 4-6 nov. 2004).

MeINER, J. W. [1781] (1971): Philosophische Sprachlehre, Leipzig, Johann Gottlob, Fac-similé de l'édition de 1781, Stuttgart-Bad Cannstat, Friedrich Frommann Verlag.

Mı́losıch, F. (1883) : Subjektlose Sätze, Wien, W. Braunmüller.

SÉRIOT, P. (2002): Une syntaxe évolutive : l'opposition verbo-nominale et le progrès de la pensée chez Potebnja, in Rousseau (éd.), 
Histoire de la syntaxe, 1870-1940 / Modèles linguistiques, XXIII, 1 .

SÉRIOT, P. (2004) : L'affaire du petit drame : filiation franco-russe ou communauté de pensée? (Tesnière et Dmitrievskij), in Slavica Occitania, 17, Toulouse, 93-118.

SÉRIOT, P. (2006): Que fait le vent quand il ne souffle pas? à paraitre in Actes du Colloque sur la structure de la proposition (Université de Lausanne, 5-7 oct. 2006).

SÉRIOT, P. \& A. BERRENDONNER (éds.): (2000, Le paradoxe du sujet. Les propositions impersonnelles dans les langues slaves et romanes / Cahiers de l'ILSL, 12. Lausanne, Unil.

TESNIÈRE, L. (1959) : Éléments de syntaxe structurale, Paris, Klincksieck. 